\title{
Digital Image Indexing Using Secret Sharing Schemes: A Unified Framework for Single-Sensor Consumer Electronics
}

\author{
Rastislav Lukac, Member, IEEE, and Konstantinos N. Plataniotis, Senior Member, IEEE
}

\begin{abstract}
This paper introduces a color filter array (CFA) image indexing approach for cost-effective consumer electronics with image capturing capability. Using a secret sharing technique, the proposed method indexes captured images directly in the single sensor digital camera, mobile phone and pocket device by embedding metadata information in the CFA domain. The metadata are used to determine ownership, capturing device identification numbers, and to provide time and location information. Additional semantic information could be added in the metadata by the end-user through the mobile phone's or pocket device's keyboard. After the metadata are embedded to the CFA image, the subsequent demosaicking step reconstructs a full color RGB image with excellent visual quality. The metadata information can be extracted from the CFA images. Alternatively, it can be recovered by the demosaicked images in personal image databases using PC software commonly available by camera manufacturers or with conventional public image database tools. The uniqueness and efficiency of the proposed approach are demonstrated here by employing a common Bayer CFA based imaging pipeline, however, the approach is suitable for other, non-Bayer CFA patterns, as well. ${ }^{l}$
\end{abstract}

Index Terms - Consumer electronics, single-sensor imaging, digital camera image processing, image databases, image indexing, metadata, secret sharing, visual cryptography, Bayer pattern, color filter array interpolation, demosaicking.

\section{INTRODUCTION}

Cost-effective consumer electronics, such as digital cameras, pocket devices, wireless phones, and imaging devices for surveillance and automotive applications capture the image scene using a single imaging sensor [1],[2]. The sensor, usually a chargecoupled device (CCD) [3] or complementary metal oxide semiconductor (CMOS) [4] sensor, is essentially a monochromatic device and thus, it should be used in conjunction with a color filter array (CFA) to capture all the three, Red-Green-Blue (RGB) primary colors at the same time [5],[6]. The CFA image constitutes a mosaic-like gray-scale image since each pixel in the array stores a single value using spectrally selective filters [7],[8]. To produce the full-color RGB image, the two missing color

The authors are with The Edward S. Rogers Sr. Department of ECE, University of Toronto, Canada.

Corresponding Author: Dr. Rastislav Lukac, Multimedia Laboratory, BA 4157, The Edward S. Rogers Sr. Department of ECE, University of Toronto, 10 King's College Road, Toronto, Ontario, M5S 3G4, Canada (email: lukacr@ieee.org, web: http://www.dsp.utoronto.ca/ lukacr)
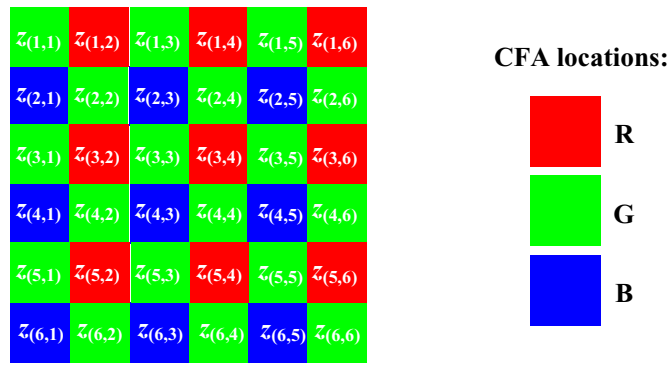

Fig. 1. Bayer CFA pattern with the GRGR phase in the first row.

components must be estimated from the adjacent pixels using the CFA interpolation or demosaicking process [7]-[9].

A number of CFAs, varying in the arrangements of the color filters and their design characteristics, have been proposed. A Bayer CFA (Fig. 1) [10] is commonly used in consumer electronic devices due to the simplicity of the subsequent demosaicking steps and for this reason is used throughout this paper. By allocating more spatial samples $(50 \%)$ to the G plane, the Bayer CFA improves the perceived sharpness of the digital image, since it is well-known that the human visual system (HVS) is more sensitive to luminance which is composed primarily of green light [9],[11].

In this paper, we introduce a new CFA data based image indexing solution, where a single-sensor captured image is connected to digital databases using embedded metadata. Depending on the consumer electronics device employed, the metadata can vary in the type and amount of the information to be processed. For example, images captured by common digital cameras can be automatically indexed using the camera's identification number, ownership information and a time stamp. In imaging enabled phones, the advanced, satellite tracking based solutions can be used to provide location stamps. Furthermore, metadata can be completed by adding semantic content through the mobile phone's or pocket device's keyboard. To unify the approach for imaging solutions, which allow for image storing in either raw (CFA) or demosaicked (RGB) formats, the proposed method indexes captured images directly in the capturing device by embedding metadata information in the CFA domain.

The indexing operations are performed through the bit-level based secret sharing concepts of [12],[13] due to their capability to perfectly reconstruct the input (secret) data using very simple implementation steps. In order to preserve the highest perceptual quality of the captured image, the proposed CFA indexer operates on the least significant bits of R and B CFA samples. By employing the indexed CFA image, the subsequent demosaicking solution produces a full color, demosaicked, image which is perceptually identical to the one reconstructed using the nonindexed, original CFA data. 
(a)
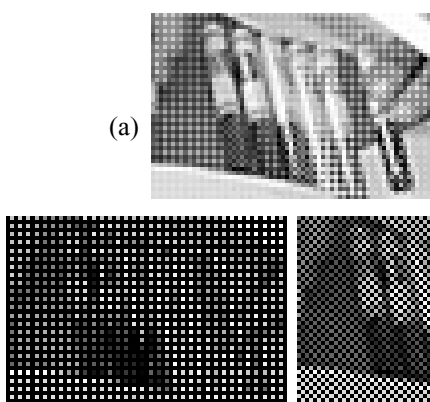

(c)

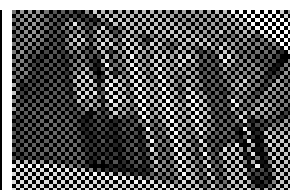

(d)

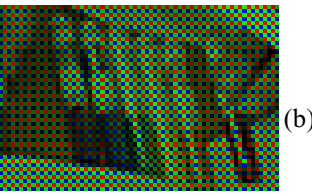

(b)

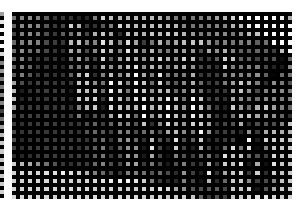

(e)

Fig. 2. Bayer CFA image: (a) raw sensor data, (b) CFA data arranged as a color image, (c) extracted samples corresponding to $R$ CFA locations, (d) extracted samples corresponding to G CFA locations, (e) extracted samples corresponding to B CFA locations.

The metadata information should be extracted from either the CFA images or the demosaicked images in personal image databases using PC software commonly available by camera manufacturers. Alternatively, conventional public image database tools such as the well-known World Wide Media eXchange (WWMX) database [14] can be used instead. Thus, the proposed approach helps to authenticate, organize and retrieve images in personal digital databases. The vast number of captured images and the exponentially growing market with image-enabled consumer electronics give us a good reason to believe that the proposed image indexing concept will be of great importance to the end-users, database software programmers, and consumer electronics manufacturers.

The rest of this paper is organized as follows. In Section II, the fundamentals of Bayer CFA based demosaicking are briefly described for completeness. The proposed secretsharing based camera image indexing solution is introduced in Section III. Motivation and design characteristics are discussed in detail. In Section IV, the proposed solution is tested using a variety of color images. Comparisons, in terms of performance, between indexed and non-indexed demosaicked images are also provided. Finally, conclusions are drawn in Section V.

\section{BAYER CFA BASED IMAGING FUNDAMENTALS}

Let us consider a digital camera solution operating on the Bayer CFA shown in Fig. 1. Due to the monochromatic nature of the sensor, the raw CFA data constitute a $K_{1} \times K_{2}$ grayscale image $z: Z^{2} \rightarrow Z$, such as the one depicted in Fig. 2a. This CFA image represents a two-dimensional matrix of integer samples $z_{(r, s)}$, with $r=1,2, \ldots, K_{1}$ and $s=1,2, \ldots, K_{2}$ denoting the image rows and columns, respectively. Based on the arrangements of color filters in the Bayer CFA, the grayscale image $z$ can be transformed to a $K_{1} \times K_{2}$ color (RGB) image $\mathbf{x}: Z^{2} \rightarrow Z^{3}$ depicted in Fig. 2b, as follows [1],[7]:

$$
\mathbf{x}_{(r, s)}= \begin{cases}{\left[z_{(r, s)}, 0,0\right]} & \text { for } r \text { odd and } s \text { even, } \\ {\left[0,0, z_{(r, s)}\right]} & \text { for } r \text { even and } s \text { odd, } \\ {\left[0, z_{(r, s)}, 0\right]} & \text { otherwise. }\end{cases}
$$
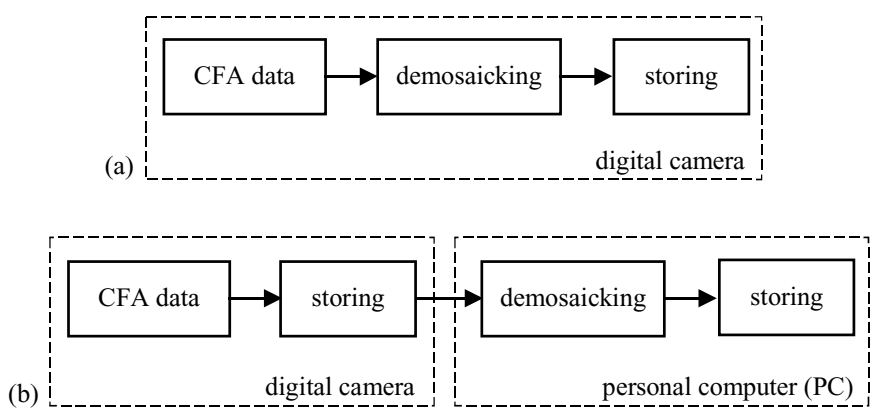

Fig. 3. Camera image processing architectures: (a) the solution with the demosaicking step performed by the digital camera, (b) the solution with the demosaicking step performed by the personal computer.

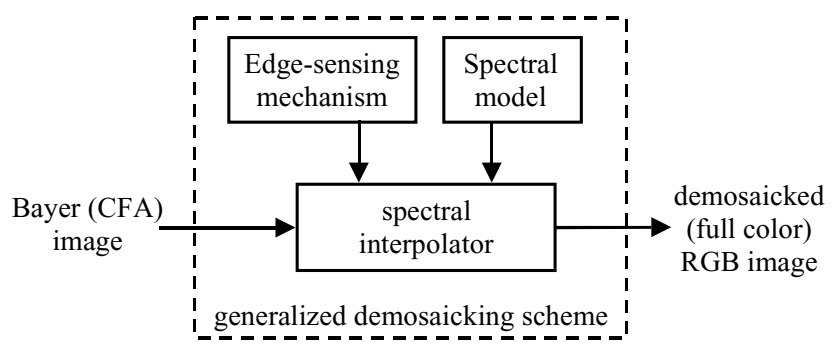

Fig. 4. Generalized demosaicking approach.

where $z_{(r, s)}$ are sensor data and $\mathbf{x}_{(r, s)}=\left[x_{(r, s) 1}, x_{(r, s) 2}, x_{(r, s) 3}\right]$ denote an RGB vector with $x_{(r, s) k}$ indicating the $\mathrm{R}(k=1), \mathrm{G}$ $(k=2)$ and $\mathrm{B}(k=3)$ component. Thus, the color image $\mathbf{x}$ represents a two-dimensional matrix of three-component samples. Visual inspection of the images depicted in Figs. 2a,b reveals that both images $z$ and $\mathbf{x}$ have a mosaic-like structure due to the fact that the spatial locations $(r, s)$ correspond to different (RGB) spectral bands, as shown in Figs. 2c-e.

The demosaicking procedure recovers missing color components from the adjacent Bayer data using an interpolator operating in the spectral (color) domain [7],[15]. As shown in Fig. 3, the demosaicking solution can be implemented in i) a conventional digital camera (Fig. 3a) which stores the demosaicked (RGB) output, or in ii) a companion personal computer (PC) which interfaces with the digital camera which stores the images in the raw CFA format (Fig. 3b). Both above processing pipelines can use the same demosaicking solution [7], however, the approach depicted in Fig. 3b allows for the utilization of sophisticated demosaicking schemes which cannot, due to their complexity, be embedded in the conventional camera image processing pipeline (Fig. 3a) which has to operate under real-time constraints.

Fig. 4 depicts the block diagram representation of a common demosaicking solution, [7]. This framework generalizes most, if not all, demosaicking schemes. As shown in Fig. 4, the characteristics of the demosaicking procedure are essentially determined by the edge-sensing mechanism and the spectral model. 


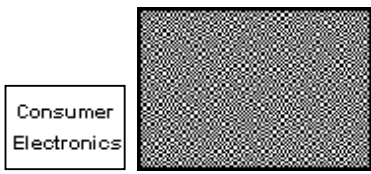

(b)

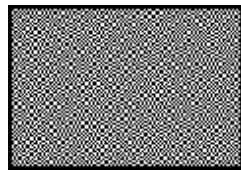

(c)

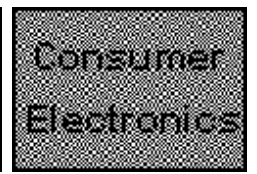

(d)
Fig. 5. A $\{2,2\}$-VSS scheme: (a) input image, (b,c) share images, (d) output, decrypted image with a processing error.

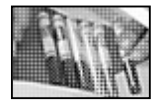

(a)

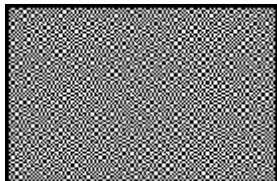

(c)

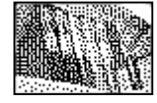

(b)

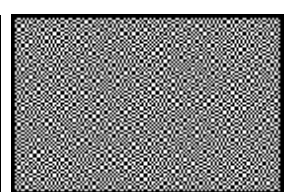

(d)

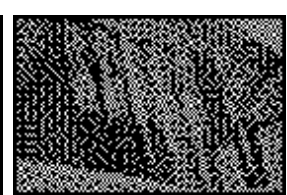

(e)
Fig. 6. A halftoning based $\{2,2\}$-VSS scheme: (a) input CFA mage, (b) halftone version of the input, (c,d) share images, (e) output, decrypted image with a processing error.

The edge-sensing mechanism is used to direct the interpolation process along edges thus preserving the sharpness and structural information of the demosaicked image. The edge sensitivity is usually controlled by parameters, such as the weighting coefficients [5],[7],[16],[17] which can be determined in an automated manner or they can be controlled by the anduser [7].

The spectral model is used to preserve the spectral correlation that exists between the color components of the natural image [11]. The use of the spectral models allows for the utilization of the essential spectral information during demosaicking and helps the process to eliminate spectral artifacts and color shifts in the demosaicked image. Following the dominant contribution of the $\mathrm{G}$ information to the human perception, a common feature in the spectral models in [15], [18]-[20] is that they incorporate RG or BG spectral characteristics into the interpolation process. The interesting reader can find additional information on both the edge-sensing mechanism and the spectral models in [7],[15].

\section{Proposed CFA IMAGE INDEXING SOLUTION}

It was already mentioned that the development of indexing tools for images that the camera captures will help to authenticate, organize and retrieve the images in both personal and public image databases. In this paper, the single-sensor captured images are indexed by adding metadata information into the image content. The use of both the processing pipelines shown in Fig. 3 necessitates the development of a universal image indexing approach. Since the only common feature of both pipelines is that they capture the image in the raw CFA format, we introduce a method which indexes captured images directly in the CFA domain. In order to design a cost-effective, real-time processing solution, the proposed here method is designed to embed the metadata information using secret sharing concepts.

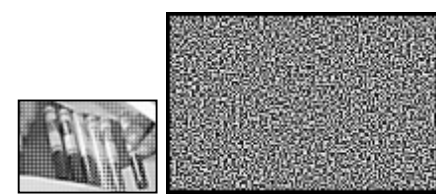

(a)

(b)

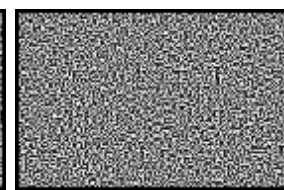

(c)

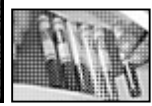

(d)
Fig. 7. A $\{2,2\}$ bit-level based secret sharing scheme: (a) input CFA mage, (b,c) share images, (d) output, decrypted CFA image identical to the input shown in (a).

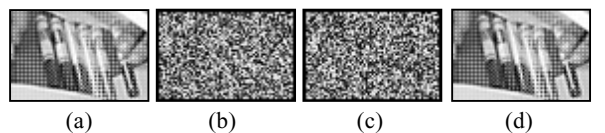

Fig. 8. An advanced $\{2,2\}$ bit-level based secret sharing scheme: (a) input CFA mage, (b,c) share images, (d) output, decrypted CFA image identical to the input shown in (a).

\section{A. Overview of the image secret sharing solutions}

Secret sharing approaches are utilized to secure transmission in multimedia and mobile public networks when the networks are used to transmit private images such as digital documents [21] and personal digital photographs [22],[23]. The existing solutions are generalized using the so-called $\{k, n\}$-scheme [21][24], which encrypts the secret image into $n$ noise-like shares which are then distributed amongst $n$ recipients. The secret image is revealed if at least $k$ shares are available for decryption. Among the numerous $\{k, n\}$-solutions, the simple $\{2,2\}$-schemes listed in the sequence are the most popular due to their use as a private-key cryptographic solution [13],[22],[25].

Fig. 5 depicts a $\{2,2\}$-visual secret sharing (VSS) scheme [24],[25]. By utilizing the transparent/frosted representation of the shares printed on transparencies, the scheme uses the properties of the HVS to force the recognition of a secret message from overlapping shares without additional computations or any knowledge of cryptography. By stacking both shares on an overhead projector the secret image is visually revealed. However, such a solution is not suitable for digital processing and due to the nature of the algorithm, the approach: i) operates on binary or binarized inputs, ii) increases the spatial dimension of both shares and output image and iii) introduces a number of visual impairments to the output image.

Fig. 6 depicts the images recorded using a halftoning based $\{2,2\}$-VSS scheme [26],[27]. In order to process gray-scale or color images via a VSS-based solution, a halftoning procedure [28] is needed in order to convert continuous-tone images into halftone images with a binary representation. The halftone image is then passed to the encryptor which produces the two binary shares. The shares are then printed on transparencies and stacked together to visually reveal the secret image. The halftoning based VSS scheme also alters the spatial resolution of the input and does not restore the input to its original quality. Moreover, the fact that the output is not recovered in digital form limits the applicability of VSS schemes in image processing applications. 


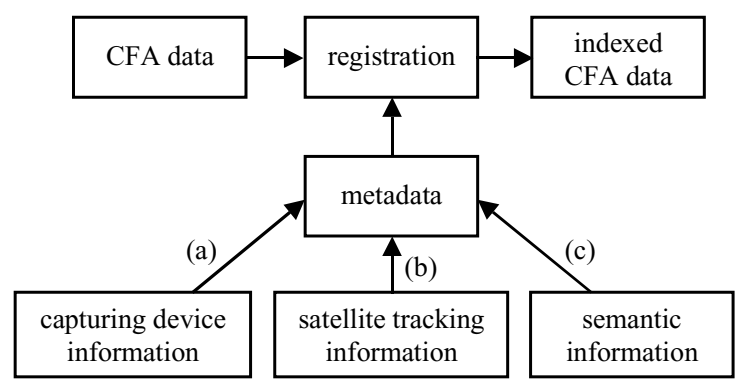

Fig. 9. CFA image indexing concept. The metadata can contains: (a) capturing device identification number, ownership and time stamps, (b) location stamps, and (c) semantic information provided by the end-user via the device's keyboard and/or microphone.

The $\{2,2\}$-secret sharing scheme in [12] operates on the bit planes of the digital input. Thus, the scheme generates the shares with a bit representation identical to the one of the input image. Visual inspection of the images shown in Fig. 7 reveals that although the input image and the produced shares have different spatial dimensions, the decryption procedure produces an output image which is identical to the input. This suggests that this private-key cryptographic solution satisfies the perfect reconstruction property [22],[23].

Finally, Fig. 8 depicts the images produced by the advanced solution of [13]. Similarly to the $\{2,2\}$-secret sharing scheme in [12], the advanced solution performs cryptographic processing on bit planes and thus, it preserves the bitrepresentation of the shares, producing an output image identical to the input image. Since the scheme encrypts each pixel into the share pixels instead of blocks of share pixels as it is done in Figs. 5-7, the spatial resolution of the shares remain unchanged during processing. In addition, the scheme recover the input image with perfect reconstruction. Therefore, the solution of [13] will be utilized in the sequence to meet the design objective of this proposal.

\section{B. Proposed CFA image indexing procedure}

The proposed CFA image indexing solution can be universally used in both the single-sensor capturing device architectures depicted in Fig. 3. As shown in Fig. 9, the CFA image indexing concept is based on metadata which can be populated with information coming from different sources such as the capturing device, a satellite navigation system, or by the end user using the device's keyboard and/or microphone. Thus, the metadata can vary in type and the amount of the information to be processed. In addition to the capturing device identification number, ownership or time stamps, which constitute the information to be automatically supplied by all single-sensor consumer electronics devices, the use of a mobile phone [29],[30], personal digital assistant (PDA) [31],[32] or other pocket-size devices [33] offers further possibilities. Through the mobile location estimation and determination tools [29],[30], a single-sensor wireless device can index the CFA data with the location stamps.
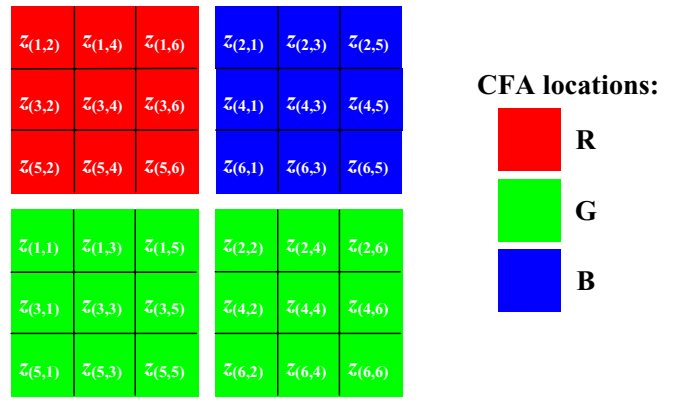

Fig. 10. Arrangement of the colored subarrays using the CFA data $z_{(r, s)}$ shown in Fig. 1.

(a)

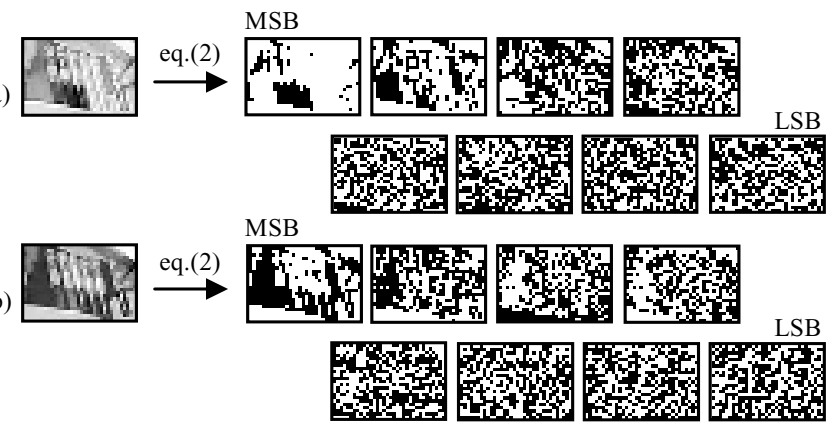

Fig. 11. Bit levels of the extracted $R$ and $B$ component of the CFA image shown in Fig. 2: (a) $R$ component, (b) $B$ component. The bit planes are listed from the MSB (left) to the LSB (right) plane.

Probably the most efficient way to represent the metadata leads to the use of a series of the bits which can be arranged as a binary image or two-dimensional binary field. Due to the binary nature of metadata information, one bit-level of the CFA image $z$ can be used to index the CFA data with the supplied metadata via the secret sharing based encryption approach of [13]. The highest perceptual quality of the captured image is thus ensured by performing the indexing operations on the LSB plane of $z$. Since the G component of the captured image contributes mostly to the essential for the HVS luminance the proposed CFA indexer should strictly affect only the $\mathrm{R}$ and $\mathrm{B}$ component of the captured data. Finally, due to the use of the $\{2,2\}$-secret sharing concepts, each metadata bit is encrypted into two bits of the indexed CFA image and thus, the maximum size of LSB based metadata information is limited (Fig. 10) to size of $K_{1} / 2 \times K_{2} / 2$ which denotes the number of $\mathrm{R}$ or B CFA locations in $z$. It should be noted that the addition of a simple end-of-file mark in metadata information allows for indexing the CFA image with trivial binary sequences. To demonstrate the processing steps performed by the proposed solution, a $K_{1} / 2 \times K_{2} / 2$ binary array of the metadata bits is used in the sequence.

Let us consider a $K_{1} / 2 \times K_{2} / 2$ binary image $m$ which represents a two-dimensional matrix of binary (metadata) samples $m_{(u, v)}$, with $u=1,2, \ldots, K_{1} / 2$ and $v=1,2, \ldots, K_{2} / 2$. Let us also consider that each integer sample $z_{(r, s)}$ of the CFA image $z$ to be indexed using the metadata image $m$ is represented by $B$ bits as follows [34]: 


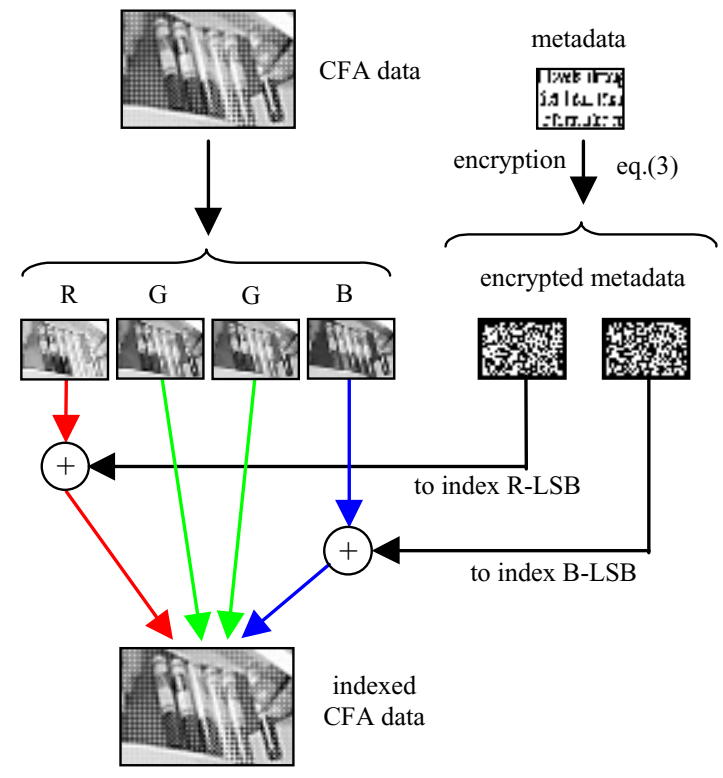

Fig. 12. Block scheme representation of the proposed CFA image indexing procedure.

$z_{(r, s)}=z_{(r, s)}^{1} 2^{B-1}+z_{(r, s)}^{2} 2^{B-2}+\ldots+z_{(r, s)}^{B-1} 2+z_{(r, s)}^{B}$

where $(r, s)$ denotes the spatial location in $z$ and $z_{(r, s)}^{b}$ indicates the bit value at the bit level $b=1,2, \ldots, B$. Using (2) the CFA image $z$ is decomposed into a series of $B$ binary images (Fig. 11), with $b=B$ denoting the LSB plane.

It is evident that a number of encryption functions can be used to combine the LSB plane with the binary metadata. To provide the most cost-effective solution, the proposed scheme performs the image indexing operation as follows:

$\left[z_{(2 u-1,2 v)}^{B}, z_{(2 u, 2 v-1)}^{B}\right]=f_{e}\left(m_{(u, v)}\right) \in \begin{cases}\{[0,1],[1,0]\} & \text { if } m_{(u, v)}=1 \\ \{[0,0],[1,1]\} & \text { if } m_{(u, v)}=0\end{cases}$

where $z_{(2 u-1,2 v)}^{B}$ and $z_{(2 u, 2 v-1)}^{B}$ denote the LSB of R or B CFA component $z_{(., .)}$, respectively. Based on the values of $u$ and $v$ which determine the spatial location $(u, v)$ of the metadata $m_{(u, v)}$ in the binary image $m$, the necessary R and B CFA samples are extracted from $z$ in a fully automated manner.

The mapping in (3) is guided by a random number generator which determines randomly the character of the indexed LSB plane of the R and B CFA components. To ensure the random nature of the encryption, the electronic noise sources [35] naturally present in the single-sensor capturing device could be used to encrypt the metadata bit $m_{(u, v)}$ via the encryption function $f_{e}($.$) in (3). To simulate such a random process, the$ conventional rand function that is built in to common programming tools, is used throughout this paper. Based on the actual value of the metadata information $m_{(u, v)}$, the image indexing process determines the indexed R and B LSB from the sets $\{[0,1],[1,0]\}$ for the high $\left(m_{(u, v)}=1\right)$ or from the sets $\{[0,0],[1,1]\}$ for the low $\left(m_{(u, v)}=0\right)$ value of $m_{(u, v)}$.

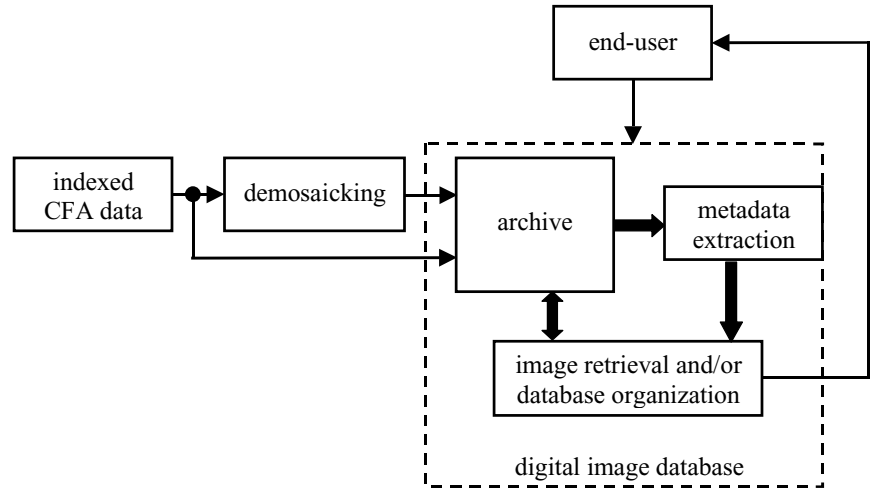

Fig. 13. Block scheme representation of the digital camera image database architecture.

Fig. 12 shows a system level diagram of the proposed CFA image indexing solution. The metadata are encrypted into two share-like binary, encrypted metadata images. Each one of the encrypted metadata images is further registered with the $\mathrm{R}$ and $\mathrm{B}$ LSB planes of the input CFA image via (3). In this way, the procedure generates an indexed CFA image, which can be stored or processed in the subsequent demosaicking procedure following the scenario depicted in Fig. 3.

\section{Proposed metadata extraction procedure}

Using the CFA image indexing operations a single-sensor captured image can be connected to a digital repository via the embedded metadata. As shown in Fig. 13, both the indexed CFA images or their demosaicked variants can be archived uniquely organized and retrieved in digital databases using the extracted metadata information.

The metadata extraction procedure recovers the original metadata bits $m_{(u, v)}$ by processing the LSB plane of the indexed CFA or demosaicked image. If the indexed CFA image $z$ is considered, following (3) the metadata extraction procedure classifies the original binary pixel $m_{(u, v)}$ as one or zero using the following operation:

$m_{(u, v)}=f_{d}\left(z_{(2 u-1,2 v)}^{B}, z_{(2 u, 2 v-1)}^{B}\right) \in \begin{cases}1 & \text { if } z_{(2 u-1,2 v)}^{B} \neq z_{(2 u, 2 v-1)}^{B} \\ 0 & \text { if } z_{(2 u-1,2 v)}^{B}=z_{(2 u, 2 v-1)}^{B}\end{cases}$

If the metadata are to be extracted from the demosaicked image, following (1) which denotes the arrangements of the original CFA data in the demosaicked image $\mathbf{x}$, the decryption function $f_{d}($.$) in (4) should be defined as follows:$

$m_{(u, v)}=f_{d}\left(x_{(2 u-1,2 v) 1}^{B}, x_{(2 u, 2 v-1) 3}^{B}\right) \in \begin{cases}1 & \text { if } x_{(2 u-1,2 v) 1}^{B} \neq x_{(2 u, 2 v-1) 3}^{B} \\ 0 & \text { if } x_{(2 u-1,2 v) 1}^{B}=x_{(2 u, 2 v-1) 3}^{B}\end{cases}$

Thus, (4) and (5) constitute a universal extraction tool for the metadata information embedded via (3). It is evident that the proposed approach recovers the original metadata allowing thus for perfect reconstruction of essential embedded information. 


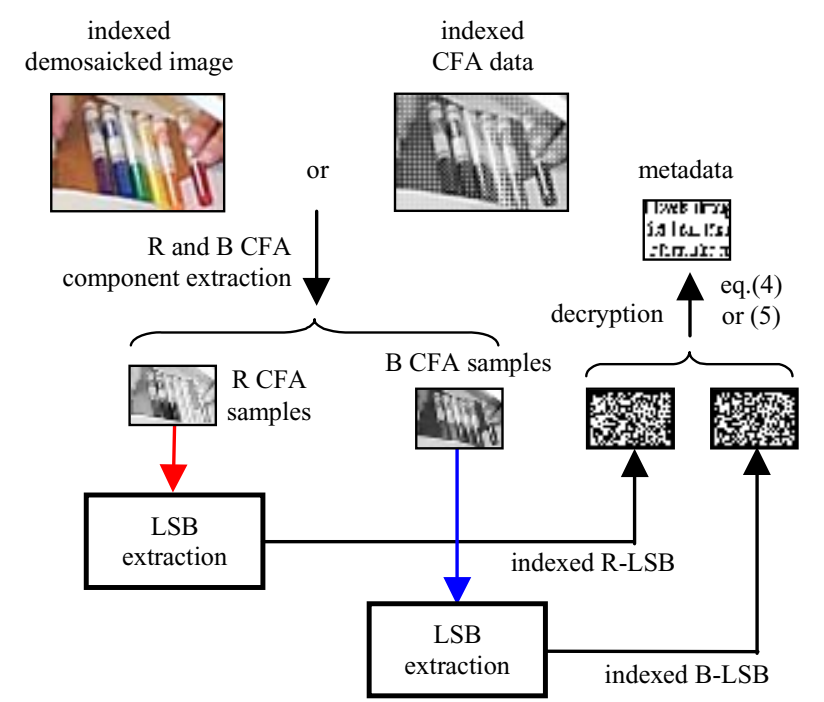

Fig. 14. Block scheme representation of the proposed metadata extraction procedure.

Fig. 14 depicts a system level diagram of the proposed metadata extraction procedure. The metadata extraction process starts with the separation of the original $\mathrm{R}$ and $\mathrm{B}$ CFA components. Since these components are not affected by the demosaicking procedure, they are present in both CFA and demosaicked images. In the next step, the $\mathrm{R}$ and $\mathrm{B}$ CFA components undergo (2) in order to extract the corresponding LSB values which are used in the sequence to decrypt the metadata bits $m_{(u, v)}$ via (4) or (5). The metadata decryption step completes the proposed extraction procedure and recovers the original metadata information.

\section{EXPERIMENTAL RESULTS}

To examine the performance of the proposed CFA image indexing solution, a number of color and binary test images have been used. Examples such as the $512 \times 512$ color images Lighthouse and Train depicted in Figs. 15a,b are used to represent the captured scenario. These test images, which vary in color appearance and complexity of the structural content (edges), have been captured using three-sensor devices and normalized to 8-bit per channel RGB representation. The $256 \times 256$ binary images Text and Shape which are used to simulate the metadata, are shown in Figs. 15c,d. The use of the metadata information in the form of a binary text image and the various geometrical shapes allows for the demonstration of the robustness of the proposed CFA indexing approach.

Please note that the objective of the experimentation presented in the sequence, is not to evaluate the performance of the demosaicking solutions. This problem was addressed, from a demosaicking [5],[7], spectral modelling [7],[15],[18] and postprocessing [18] point of view, in our previous works. In this section, we intent to demonstrate that the proposed CFA indexing solution preserves the perceptual quality of the CFA and most importantly, that our method does not affect the perceptual quality of the demosaicked images.

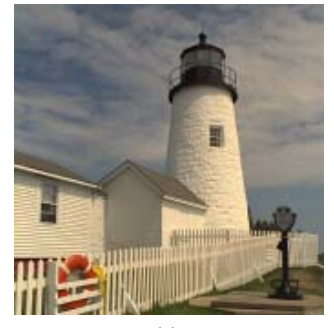

(a)

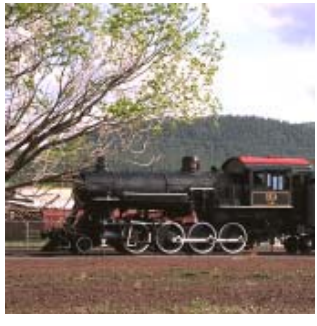

(b)

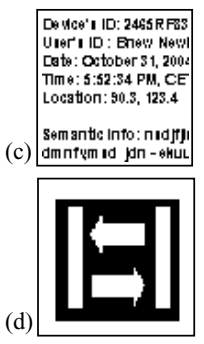

Fig. 15. Test color images $(a, b)$ and the binary metadata images $(c, d)$. (a) Lighthouse, (b) Train, (c) Text, (d) Shape.

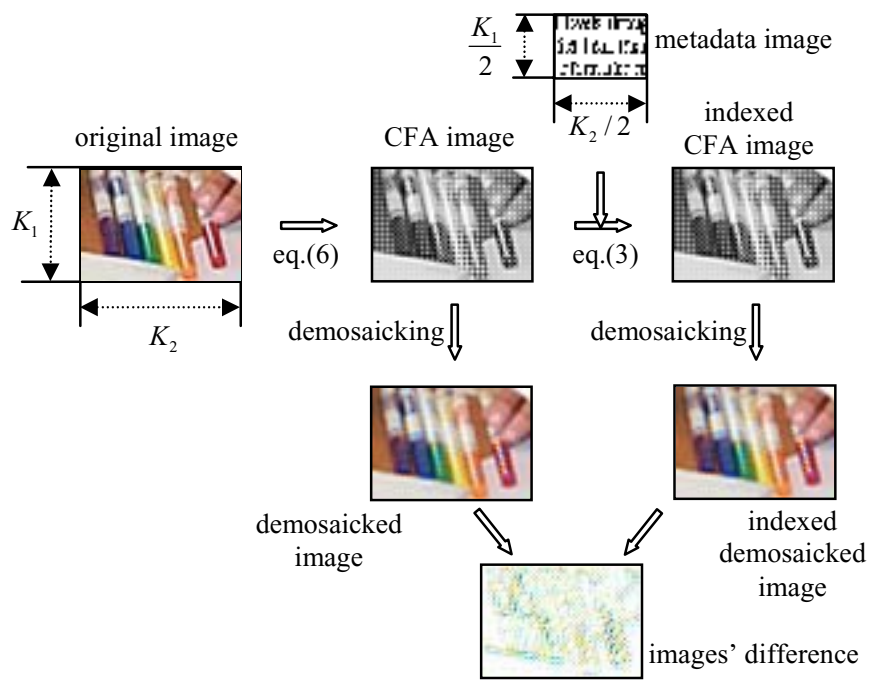

Fig. 16. Evaluation procedure.

Fig. 16 visualizes the evaluation procedure used in this paper. A set of test images, such as those shown in Fig. 15, is constituted. Mosaic versions of the $K_{1} \times K_{2}$ original color images $\mathbf{0}$ are created by discarding color information in a GRGR phased Bayer CFA filter (Fig. 1) resulting in the CFA image $z$ with pixels $z_{(r, s)}$ defined as follows [5],[7]:

$z_{(r, s)}= \begin{cases}o_{(r, s) 1} & \text { for } r \text { odd and } s \text { even, } \\ o_{(r, s) 3} & \text { for } r \text { even and } s \text { odd, } \\ o_{(r, s) 2} & \text { otherwise. }\end{cases}$

where $o_{(r, s) k}$ denotes the $\mathrm{R}(k=1), \mathrm{G}(k=2)$ and $\mathrm{B}(k=3)$ component of the original color vector $\mathbf{o}_{(r, s)}=\left[o_{(r, s) 1}, o_{(r, s) 2}, o_{(r, s) 3}\right]$ with $r=1,2, \ldots, K_{1}$ and $s=1,2, \ldots, K_{2}$. The indexed version of the CFA image $z$ is produced via the proposed CFA image solution (3) with the $K_{1} / 2 \times K_{2} / 2$ binary image $m$, which is formed by the metadata bits $m_{(u, v)}$. The demosaicked and indexed demosaicked images are obtained from $\mathbf{x}$ applying the identical demosaicking solution to process the non-indexed ("original") and indexed CFA image. The difference in performance between the two approaches is measured using both objective and subjective criteria, by comparing the quality of the demosaicked images against the indexed demosaicked images. 
TABLE I

\begin{tabular}{lcccc}
\multicolumn{4}{l}{ COMPARISON OF THE METHODS USING THE LIGHTHOUSE IMAGE } \\
\hline \hline $\begin{array}{c}\text { Metadata } \\
\text { Image }\end{array}$ & Method & MAE & MSE & NCD \\
\hline \multirow{3}{*}{ Text } & BI & 0.255 & 0.260 & 0.00435 \\
& KA & 0.431 & 0.604 & 0.00514 \\
& CCA & 0.299 & 0.306 & 0.00345 \\
\hline \multirow{3}{*}{ Shape } & BI & 0.257 & 0.260 & 0.00430 \\
& KA & 0.430 & 0.604 & 0.00507 \\
& CCA & 0.302 & 0.309 & 0.00341 \\
\hline
\end{tabular}

TABLE II

COMPARISON OF THE METHODS USING THE TRAIN IMAGE

\begin{tabular}{ccccc}
\hline \hline $\begin{array}{c}\text { Metadata } \\
\text { Image }\end{array}$ & Method & MAE & MSE & NCD \\
\hline \multirow{3}{*}{ Text } & BI & 0.286 & 0.290 & 0.00566 \\
& KA & 0.460 & 0.824 & 0.00710 \\
& CCA & 0.331 & 0.344 & 0.00473 \\
\hline \multirow{2}{*}{ Shape } & BI & 0.286 & 0.290 & 0.00555 \\
& KA & 0.458 & 0.715 & 0.00697 \\
& CCA & 0.332 & 0.345 & 0.00464 \\
\hline
\end{tabular}

The mean absolute error (MAE), the mean square error (MSE) and the normalized color difference (NCD) criterion are used here to objectively measure the difference between non-indexed and indexed demosaicked images. These objective criteria are commonly used in the image processing community and the interesting reader can find their definition in our previous works, e.g. [5],[7]. It should be emphasized that the MAE and MSE criteria are used to measure the difference between the two images in the RGB color space. To measure this difference in units corresponding to human perception, the NCD criterion, defined in the perceptually uniform CIE LUV color space is used.

To perform the demosaicking step, the following three algorithms, namely the bilinear interpolation (BI) [6], the Kimmel's algorithm (KA) [17] and the correlation-correction approach (CCA) [5] have been used. The above set of the demosaicking solutions includes the simplest BI scheme, the sophisticated KA scheme, and the CCA scheme which is one of the most powerful demosaicking solutions.

Tables I and II summarize the objective results obtained using the test images shown in Fig. 15. It can be observed that there is a negligible difference between the non-indexed and indexed demosaicked images. Moreover, the use of statistically different color images as well as the use of different metadata images does not change the results. This suggests that the proposed CFA image indexing approach is sufficiently robust to handle different imaging scenarios and different types of metadata information

Fig. 17 allows for the visual comparisons of the "original" and indexed CFA images. From the presented examples it is evident that there is no difference between non-indexed and indexed CFA data from a human visual perception point of view. This is due to the fact that: i) the CFA data constitute a mosaic of the gray-scale intensities corresponding to different spectral bands, and ii) the indexing was performed on the LSB plane of the chrominance (R, B) signals and not on the $G$ channel that dominates the luminance information.

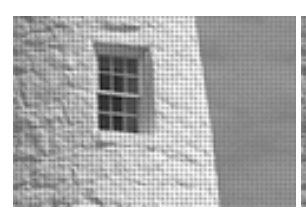

(a)

(b)

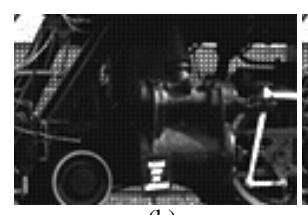

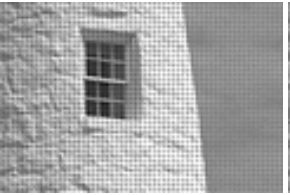

(c)

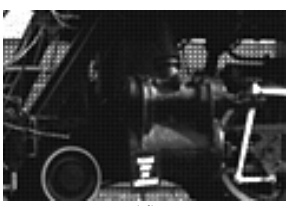

(d)

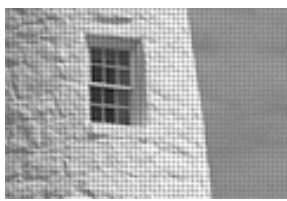

(e)

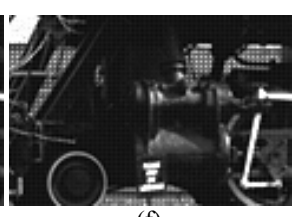

(f)
Fig. 17. Cropped areas of the CFA images corresponding to the image Lighthouse $(a, c, e)$ and Train $(b, d, f)$. The images shown in $(a, b)$ contains non-indexed data, while $(c, d)$ and $(e, f)$ correspond to the data indexed via the binary image Text and Shape, respectively.

Since most captured images are intended for human inspection in the full color, RGB format, Figs. 18-19 facilitate the visual comparison of the demosaicked images. The results indicate that it is impossible to visually determine which demosaicked image contains the embedded metadata. Built on the excellent design characteristics of the proposed CFA image indexing solution, the subsequent demosaicking step never introduces additional impairments or degradation of the visual quality other than those introduced by the demosaicking solution while it operates on the non-indexed CFA data.

Finally, the proposed solution is computationally efficient since the execution of the developed indexing tool on a PC with an Intel Pentium IV 2.40 GHz CPU, 512 MB RAM, Windows $\mathrm{XP}$ operating system and MS Visual $\mathrm{C}^{++} 5.0$ programming environment, took (on average) 0.086 (in the indexing phase) and $0.040 \mathrm{sec}$ (in the extraction phase) per a $512 \times 512$ input image with and a $256 \times 256$ metadata image.

In the summary, the following conclusions can be drawn: i) the use of proposed image indexing concept preserves both spectral and spatial information of the captured image, ii) the proposed indexing solution does not introduce any side effect nor decrease the measured and/or visual quality of the captured image, and iii) the approach is sufficiently robust and computationally efficient.

\section{Conclusion}

A unique CFA image indexing approach was introduced. The approach utilizes powerful secret sharing concepts to embed metadata into the single-sensor captured image. By processing the LSB plane of the R and B CFA components, the metadata information is, in an imperceivable to humans way, embedded in the CFA domain. The extraction procedure recovers the original metadata from either indexed CFA or demosaicked image. The extracted metadata can be used: i) as security feature, ii) to facilitate indexing and retrieval in personal imaging databases, iii) to prove ownership of produced digital data and equipment, and iv) for forensic examination of information. The efficiency and versatility of the proposed framework make our approach ideal for a new generation of consumer electronics. 
(a)
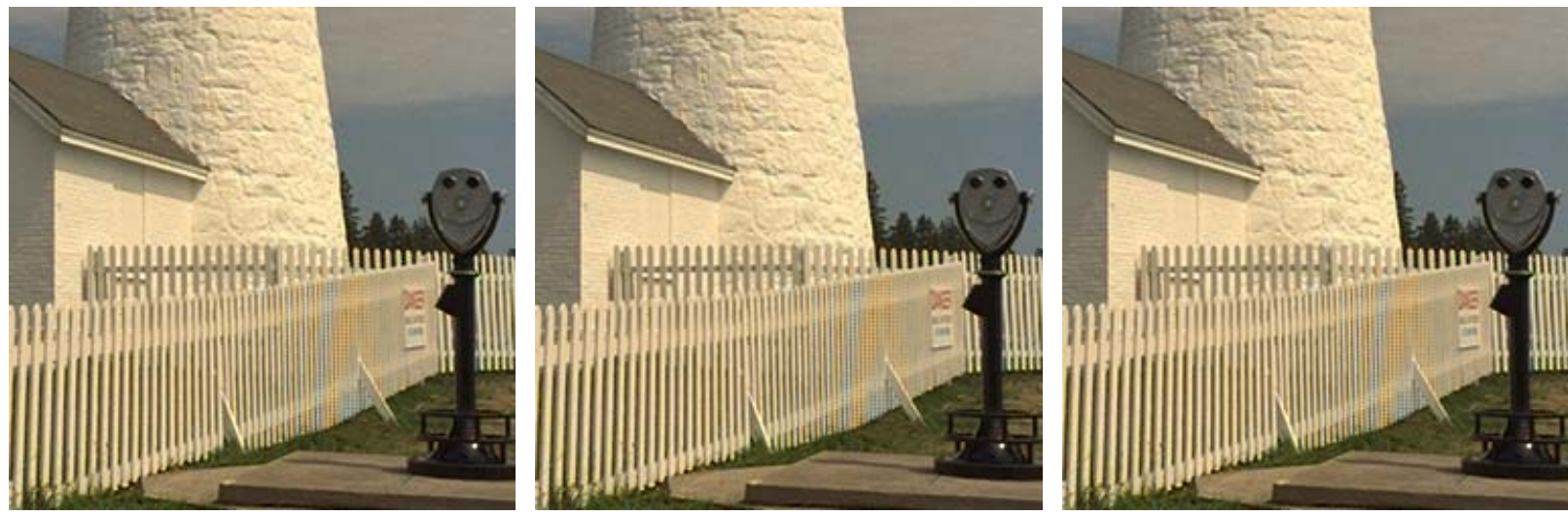

(b)
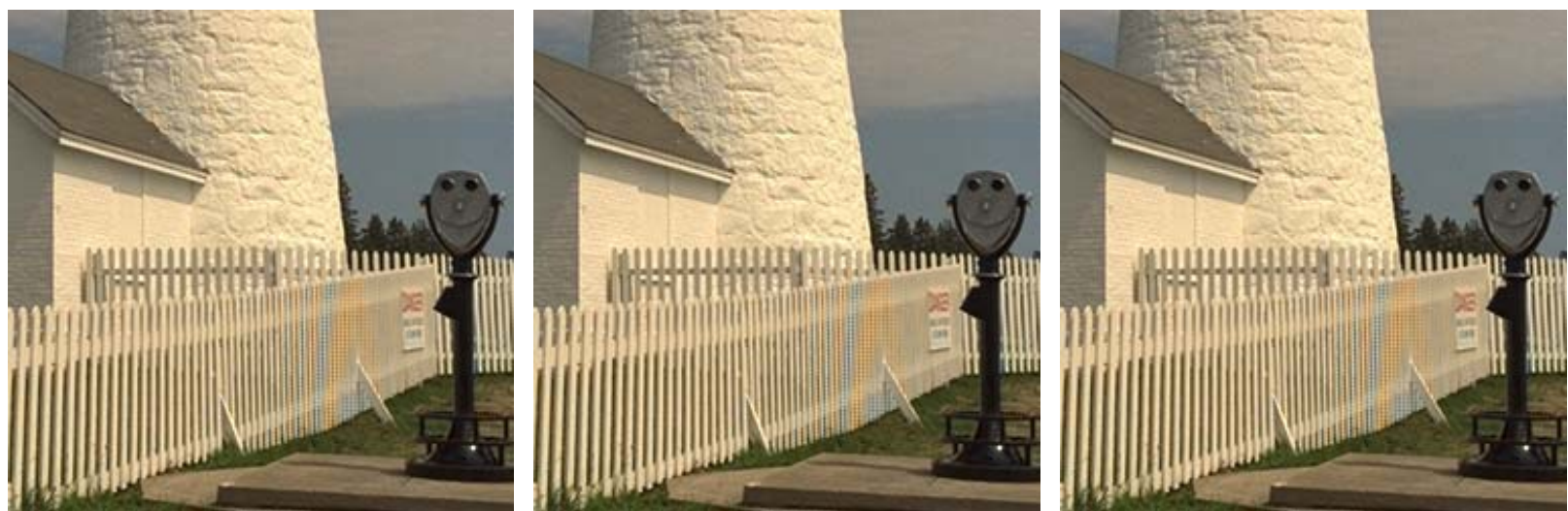

Fig. 18. Cropped areas of the demosaicked images Lighthouse: (a) KA, (b) CCA; with the left, middle and right column corresponding to nonindexed data, data indexed using the binary image Text, and data indexed using the binary image Shape

(a)
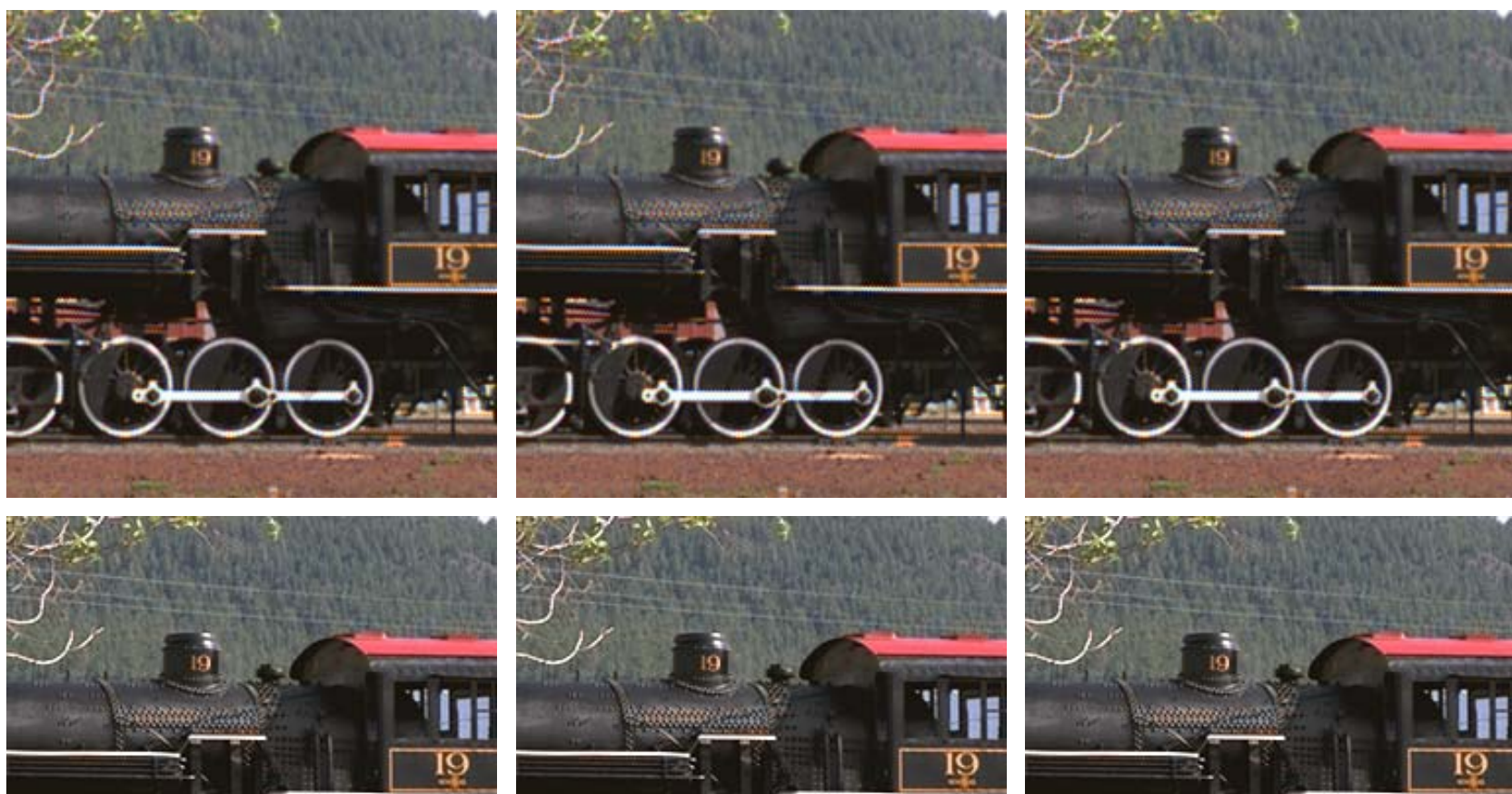

(b)
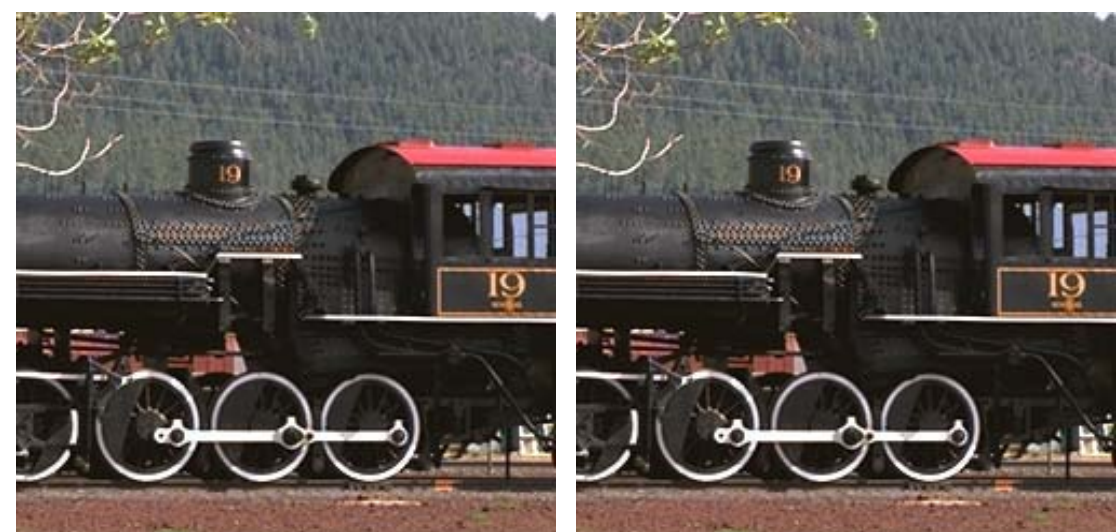

Fig. 19. Cropped areas of the demosaicked images Train: (a) BI, (b) CCA; with the left, middle and right column corresponding to non-indexed data, data indexed using the binary image Text, and data indexed using the binary image Shape 


\section{REFERENCES}

[1] R. Lukac, K. Martin, and K. N. Plataniotis, "Digital camera zooming based on unified CFA image processing steps," IEEE Transactions on Consumer Electronics, vol. 50, no. 1, pp.15-24, February 2004.

[2] K. Parulski and K.E. Spaulding, "Color image processing for digital cameras," in Digital Color Imaging Handbook, (eds.) G. Sharma, CRC Press, Boca Raton, FL., pp.728-757, 2002.

[3] P.L.P. Dillon, D.M. Lewis, and F.G. Kaspar, "Color imaging system using a single CCD area array," IEEE Journal of Solid-State Circuits, vol. 13, no. 1, pp. 28-33, February 1978 .

[4] T. Lule, S. Benthien, H. Keller, F. Mutze, P. Rieve, K. Seibel, M. Sommer, and M. Bohm, "Sensitivity of CMOS based imagers and scaling perspectives," IEEE Transactions on Electron Devices, vol. 47, no. 11 , pp. $2110-2122$, November 2000

[5] R. Lukac, K. N. Plataniotis, D. Hatzinakos, and M. Aleksic, "A novel cost effective demosaicing approach," IEEE Transactions on Consumer Electronics, vol. 50, no. 1, pp. 256-261, February 2004.

[6] R. Ramanath, W. E. Snyder, G. L. Bilbro, and W. A. Sander III, "Demosaicking methods for Bayer color arrays," Journal of Electronic Imaging, vol. 11, no. 3, pp. 306-315, July 2002.

[7] R. Lukac, and K.N. Plataniotis, "Data-adaptive filters for demosaicking: a framework," IEEE Transactions on Consumer Electronics, vol.. 51, no. 2, May 2005.

[8] S. C. Pei and I.K. Tam, "Effective color interpolation in CCD color filter arrays using signal correlation," IEEE Trans. Circuits and Systems for Video Technology, vol. 13, no. 6, pp. 503-513, June 2003.

[9] B. Gunturk, Y. Altunbasak, and R. Mersereau, "Color plane interpolation using alternating projections," IEEE Transactions on Image Processing, vol. 11, no.9, pp. 997-1013, September 2002.

[10] B. E. Bayer, "Color imaging array," U.S. Patent 3971 065, July 1976

[11] K. N. Plataniotis and A. N. Venetsanopoulos, Color Image Processing and Applications. Springer Verlag, Berlin, 2000.

[12] R. Lukac and K.N Plataniotis, "Colour image secret sharing," IEE Electronics Letters, vol. 40, no. 9, pp. 529-530, April 2004.

[13] R. Lukac and K.N. Plataniotis, "A cost-effective private-key cryptosystem for color image encryption," Lecture Notes in Computer Science, vol. 3514, pp. 687-694, May 2005.

[14] K. Toyama, R. Logan, A. Roseway, and P. Anandan, "Geographic location tags on digital images," in Proc. ACM International Conference on Multimedia in Berkeley, USA, pp. 156-166, Nov. 2003.

[15] R. Lukac, and K. N. Plataniotis, "Normalized color-ratio modelling for CFA interpolation," IEEE Transactions on Consumer Electronics, vol. 50, no. 2, pp. 737-745, May 2004.

[16] W. Lu and Y. P. Tang, "Color filter array demosaicking: new method and performance measures," IEEE Transactions on Image Processing, vol. 12, no. 10, pp. 1194-1210, October 2003.

[17] R. Kimmel, "Demosaicing: image reconstruction from color CCD samples," IEEE Transactions on Image Processing, vol. 8, no. 9, pp. 1221-1228, September 1999.

[18] R. Lukac, K. Martin, and K. N. Plataniotis, "Demosaicked image postprocessing using local color ratios," IEEE Transactions on Circuit and Systems for Video Technology, vol. 14, no. 6, pp. 914-920, June 2004.

[19] D. R. Cok, "Signal processing method and apparatus for producing interpolated chrominance values in a sampled color image signal," US Patent 4642 678, February 1987.

[20] J. Adams, "Design of practical color filter array interpolation algorithms for digital cameras," Proc. SPIE, vol. 3028, pp. 117-125, Feb. 1997.

[21] R. Lukac and K.N. Plataniotis, "Document image secret sharing using bit-level processing," in Proc. International Conference on Image Processing (ICIP'04) in Singapore, pp.2893-2896, October 2004.

[22] R. Lukac and K.N. Plataniotis, "Image representation based secret sharing," Communications of the CCISA, Special Issue on Image Secret Sharing, vol. 11, no. 2, pp. 103-114, April 2005.

[23] R. Lukac and K.N. Plataniotis, "Bit-level based secret sharing for image encryption.," Pattern Recognition, vol. 38, no. 5, pp. 767-772, May 2005.

[24] M. Naor and A. Shamir, "Visual cryptography," Lecture Notes in Computer Science, vol. 950, pp. 1-12, 1994.
[25] G. Ateniese, C. Blundo, A. de Santis, and D.R. Stinson, "Visual cryptography for general access structures," Information and Computation, vol. 129, no. 2, pp. 86-106, September 1996.

[26] C.C. Lin and W.H. Tsai, "Visual cryptography for gray-level images by dithering techniques," Pattern Recognition Letters, vol. 24, no. 1-3, pp. 349-358, January 2003.

[27] J.C. Hou, "Visual cryptography for color images," Pattern Recognition, vol. 36, no. 7, pp. 1619-1629, July 2003.

[28] R.A. Ulichney, "Dithering with blue noise," Proceedings of the IEEE, vol. 76, no. 1, pp. 56-79, January 1988

[29] M. McGuire, and K.N. Plataniotis, "Dynamic model-based filtering for mobile terminal location estimation," IEEE Transactions on Vehicular Technology, vol. 52, no. 4, pp. 1012-1031, July 2003.

[30] Y. Zhao, "Standardization of mobile phone positioning for 3G systems," IEEE Communications Mag., vol. 40, no. 7, pp. 108-116, July 2002.

[31] D. Starobinski, A. Trachtenberg, and S. Agarwal, "Efficient PDA synchronization," IEEE Transactions on Mobile Computing, vol. 2, no. 1, pp. 40-51, Jan.-March 2003.

[32] J.W. Bruce, M.A. Gray, and R.F. Follett, "Personal digital assistant (PDA) based I2C bus analysis," IEEE Transactions on Consumer Electronics, vol. 49, no. 4, pp. 1482-1487, November 2003.

[33] D. Verkest, "Machine chameleon - handheld devices," IEEE Spectrum, vol. 40, no. 12, pp. 41-46, December 2003.

[34] C.S. Burrus, "Digital filter structures described by distributed arithmetic," IEEE Transactions on Circuits and Systems, vol. 24, no. 12, pp. 674-680, December 1977.

[35] C.S. Petrie and J.A. Connelly, "A noise-based IC random number generator for applications in cryptography," IEEE Trans. on Circuits and Systems - CAS-I, vol. 47, no. 5, pp. 615-621, May 2000.

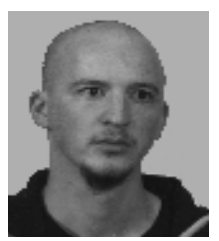

Rastislav Lukac received the M.S. (Ing.) and Ph.D. degrees in Telecommunications from the Technical University of Kosice, Slovak Republic in 1998 and 2001, respectively. From February 2001 to August 2002 he was an Assistant Professor at the Department of Electronics and Multimedia Communications at the Technical University of Kosice. Since August 2002 he is a Researcher in Slovak Image Processing Center in Dobsina, Slovak Republic. From January 2003 to March 2003 he was a Postdoctoral Fellow at the Artificial Intelligence \& Information Analysis Lab at the Aristotle University of Thessaloniki, Greece. Since May 2003 he has been a Post-doctoral Fellow with the Edward S. Rogers Sr. Department of Electrical and Computer Engineering at the University of Toronto in Toronto, Canada. His research interests include digital camera image processing, microarray image processing, multimedia security, and nonlinear filtering and analysis techniques for color image $\&$ video processing.

Dr. Lukac is a Member of the IEEE, a Member of the IEEE Circuits and Systems, IEEE Consumer Electronics, and IEEE Signal Processing Societies. He serves as a Technical Reviewer for various scientific journals and he participates as a Member of numerous International Conference Committees. In 2003 he was awarded the NATO/NSERC Science Award.

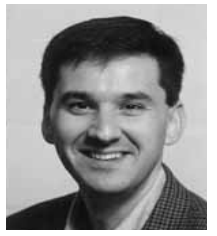

Konstantinos N. Plataniotis received the B. Engineering degree in Computer Engineering from the Department of Computer Engineering and Informatics, University of Patras, Patras, Greece in 1988 and the M.S and $\mathrm{Ph} . \mathrm{D}$ degrees in Electrical Engineering from the Florida Institute of Technology (Florida Tech), Melbourne, Florida in 1992 and 1994 respectively. He was affiliated with the Computer Technology Institute (C.T.I), Patras, Greece from 1989 to 1991. From August 1997 to June 1999 he was an Assistant Professor with the School of Computer Science at Ryerson University. He is currently an Assistant Professor at the Edward S. Rogers Sr. Department of Electrical \& Computer Engineering where he researches and teaches adaptive systems and multimedia signal processing.

Dr. Plataniotis is a Senior Member of IEEE, a past member of the IEEE Technical Committee on Neural Networks for Signal Processing. He was the Technical Co-Chair of the Canadian Conference on Electrical and Computer Engineering (CCECE) 2001, and CCECE 2004. 